\title{
The effect of pinholes of different sizes on visual acuity under different refracting states and ambi- ent lighting conditions*
}

\author{
Abdul N*, Meyer N*, van Bosch $\mathbf{M}^{*}$, van Zyl A*, Viljoen $\mathrm{M}^{*}$ and Carlson AS** \\ **Department of Optometry, University of Johannesburg, PO Box 524, Auckland Park, 2006 South \\ Africa \\ $* *<$ acarlson@uj.ac.za>
}

Received 18 February 2008; revised version accepted 6 March 2009

\begin{abstract}
Purpose: The purpose of this pilot study was to investigate the effect of different size pinholes on visual acuity (VA) at near $(0.4 \mathrm{~m})$ while various factors were altered. The alterations made involved accommodation and illumination.

Method: Four subjects were selected and their ages ranged from 7 to 14 years. Subjective refractions were performed and then near visual acuities were measured at $0.4 \mathrm{~m}$ under two different lighting conditions (460 lux and 1 lux) by adding stigmatic (or spherical) lenses of positive and negative powers in front of pinholes of diameters $1.5 \mathrm{~mm}$ and $2 \mathrm{~mm}$. Results: The results showed that there was an improvement in VA in most, however, the size of the pinhole played a minor role. Low illumination did have a drastic effect on our results not only by decreasing the amount of lenses used for near that
\end{abstract}

gave more positive results, but also when the pinhole was placed in front of the subjects. According to Borish, the pinhole does improve VA by a straight forward process of elimination produced by spherical aberrations of the eye. However, this did not seem to be the case in this study under conditions of low illumination.

Conclusion: The pinhole effect offers improvement in VA at near by reducing the amount of blur presented to the retina. This study showed that all subjects had an improvement in VA of at least two lines in high illumination. Pinhole size did not alter the results sufficiently thus suggesting that a pinhole, no matter the size, should increase the VA. However this was not the case when illumination was decreased.

Key words: Pinhole, blur circles, illumination, visual acuity

\section{Introduction}

Many people experience an improvement in their visual acuity (VA) with pupil constriction in bright light conditions due to the pinhole effect. This implies that when the pupil is small clinical irregularities of the eye are less significant. Residual refractive errors, para-central corneal scars, irregular astigmatism, eccentric opacification of the posterior lens capsule and non-cortical central cataracts are examples of clinical irregularities of the eye. The smaller the pupil, the less

\footnotetext{
* 4th year students; research project performed as part of the requirements for the course Optometry 3.

** Research supervisor
} 
defocus occurs from spherical aberration (the pinhole effect $)^{1}$. This is a single reason why subjects with large pupils, either from dilation or other reasons may see "ghosting" around an otherwise sharp image. ${ }^{2}$ Subjects whose VA cannot be improved to $20 / 20$ with pinhole are referred to as having non-refractive errors and may require referral to an ophthalmologist ${ }^{3}$.

In a clinical study done by Borish ${ }^{1}$, it was found that the most effective pinhole varied in diameter between $0.94 \mathrm{~mm}$ and $1.75 \mathrm{~mm}$. When changing the diameter of the pinhole from $1 \mathrm{~mm}$ to $0.2 \mathrm{~mm}$ it caused the print to be too blurred to be distinguished and when the aperture was even further reduced it caused the page to assume a uniform greyish cast ${ }^{1}$.

\section{Effects of the near synkinesis on pinhole}

The eye of a young person can quickly change its optical power by altering the shape of the crystalline lens. The change of power is called accommodation and is measured in dioptres (the inverse of the focal length measured in meters). The amplitude of accommodation is the dioptric difference between the near and far points of vision ${ }^{4}$. It declines at approximately $0.25 \mathrm{D}$ per year ${ }^{5}$. When one focuses on a near object activation of accommodation, convergence, eye movements as well as pupil constriction takes place. Accommodation causes an increase in the optical power of the eye to facilitate focus on the near object while convergence eye movements direct the eyes towards the near object. Pupil constriction, which is also part of the near synkinesis, increases the depth of focus and will thus assist accommodation in providing a clear image of the near object on the retina. Depth-of-focus of the eye is the range of focusing errors where the image appears not to have changed in contrast, clarity or form ${ }^{6}$. This provides important information of the range of clear vision where different monocular depth cues like target size, blur and proximal information are present under natural viewing conditions ${ }^{7}$. The depth of focus of the eye can be altered by spherical aberration of the human eye, which changes as a function of the level of accommodation. Studies have shown that spherical aberration becomes more positive with far accommodation and negative with near accommodation. Thus, this implies that the depth of focus increases towards the direction of intermediate focal distance ${ }^{8}$. There are a few controversies regarding whether accommodation is the foremost factor contributing to the pupil response at near given that it has also been suggested that pupil constriction may be the consequence of other factors such as target misalignment and proximal cues such as size, fusional vergence and near effort ${ }^{9}$.

The purpose of this preliminary pilot study was to investigate the effects of different sized pinholes on proximal VA at $0.4 \mathrm{~m}$ while various factors were altered. The alterations involved accommodation and illumination. The accommodative response was included in this study for the following reason; the level of accommodation affects the amount of spherical aberrations of the human eye and the pinhole is used to eliminate these spherical aberrations.

\section{Methods}

Four subjects were selected for participation in this study. Their ages ranged from 7 years and 10 months to 12 years and 3 months. The effects of various factors were investigated in this study including the effect of adding positive and negative stigmatic lenses as well as the effect of illumination on the perception of letters at $40 \mathrm{~cm}$ without and then with pinholes of diameter of 1 and $1.5 \mathrm{~mm}$ respectively. Each subject was tested in the afternoon (after school) on different days over a period of two weeks. All the tests on each subject were completed on the same day. The full set of measurements per subject took about 45-60 minutes.

Subjective refractions were measured on both eyes of each subject first and then their amplitudes of accommodation were measured using the negative lens method (\#19) through a phoropter, viewing the standard Snellen near acuity chart. The expected amplitudes were determined by using the following mathematical equation: $A_{\exp }=15-0.25$ Age. The compensated distance VA for all subjects was $6 / 6$ and the actual amplitudes ranged from 8.25 D to $10.75 \mathrm{D}$. For this study, only the right eyes were used.

Illumination was measured using a Sekonic Lumi Model 246 illuminometer which measures the amount of light emitted off the chart used at $0.4 \mathrm{~m}$. Two specific illuminations were used in this experiment, high (460 lux) and low illumination (1 lux). The various illuminations were obtained with the use of a rheo- 
stat on the light switch that could be adjusted to the illumination required. The VA of each subject was measured under these lighting conditions while lenses of positive power were added in $1 \mathrm{D}$ steps with the aim of relaxing accommodation. While this was done the Lighthouse Near Acuity Chart was first viewed without a pinhole and then through the pinholes with diameters of $1.5 \mathrm{~mm}$ and $2 \mathrm{~mm}$ respectively with the purpose of seeing the effect that these pinholes would have on VA. Lenses were added until a VA of $6 / 60$ or less was obtained. After this was done the exercise was repeated with lenses of negative power, thus stimulating accommodation.

The conditions were kept constant during the measurements for all the subjects. The conditions included all participants being tested in the same examination room thus maintaining the identical setting to avoid external distractions and the placement of the pinhole was always in the back part of the trial frame while the lenses were added in the front.

\section{Results and Discussion}

Table 1 shows the four subjects' ages, subjective refractions, expected and actual amplitudes of accommodation. Similar results were shown by all subjects so only results for Subject 1 will be included here in Tables 2 to 5. Table 2 shows the effect of lenses of positive power on VA for Subject 1 viewing a Lighthouse Near Acuity chart at $40 \mathrm{~cm}$ without a pinhole and through pinholes of diameter $1.5 \mathrm{~mm}$ and $2 \mathrm{~mm}$ respectively with the target illumination at 460 lux while Table 3 shows the effects with the target illuminated at 1 lux. Tables 4 and 5 show a similar effect for Subject 1 but here lenses of negative power were used. Figures 1 to 12 show the results for all the subjects for the various experimental conditions.

Table 1. Ages for the four subjects and their subjective refractions, expected and actual amplitudes of accommodation are included.

\begin{tabular}{|l|l|l|l|l|}
\hline Subject Age (yrs) & Age (yrs) & \#7A: OD only & Expected amplitude & Actual amplitude \\
\hline 1 & 12.25 & P1 $/-0.25 \times 75$ & $12.00 \mathrm{D}$ & $9 \mathrm{D}$ \\
\hline 2 & 9.45 & $+0.75 /-0.25 \times 30$ & $12.75 \mathrm{D}$ & $9.25 \mathrm{D}$ \\
\hline 3 & 7.83 & $-0.75 \mathrm{D}$ & $13.25 \mathrm{D}$ & $10.25 \mathrm{D}$ \\
\hline 4 & 9.67 & $+1.25 /-0.75 \times 180$ & $12.75 \mathrm{D}$ & $8.25 \mathrm{D}$ \\
\hline
\end{tabular}

Table 2. Effect of positive lenses on VA for Subject 1 viewing a Lighthouse Near Acuity chart at $40 \mathrm{~cm}$ without a pinhole and through a pinhole with a $1.5 \mathrm{~mm}$ and $2 \mathrm{~mm}$ diameter respectively while the target was illuminated at 460 lux. (Under certain conditions the letters on the chart were not recognizable or visible and were recorded as LNV meaning Letters Not Visible.)

\begin{tabular}{|l|l|l|l|l|l|l|}
\hline Lenses & $\begin{array}{l}\text { VA without } \\
\text { pinhole }\end{array}$ & M Notation & $\begin{array}{l}\text { VA with pinhole } \\
1.5 \mathrm{~mm}\end{array}$ & M Notation & $\begin{array}{l}\text { VA with pinhole } \\
2 . \mathrm{mm}\end{array}$ & M Notation \\
\hline \#7A & $6 / 6$ & 0.40 & $6 / 6$ & 0.40 & $6 / 6$ & 0.40 \\
\hline \#7A+1.D & $6 / 6$ & 0.40 & $6 / 6$ & 0.40 & $6 / 6$ & 0.40 \\
\hline \#7A+2.D & $6 / 6$ & 0.40 & $6 / 6$ & 0.40 & $6 / 6$ & 0.40 \\
\hline \#7A+3 D & $6 / 7.5$ & 0.50 & $6 / 7.5^{+2}$ & $0.50^{+2}$ & $6 / 7.5$ & 0.50 \\
\hline \#7A+4 D & $6 / 9$ & 0.63 & $6 / 7.5$ & 0.50 & $6 / 9^{+2}$ & $0.63^{+2}$ \\
\hline \#7A+5.D & $6 / 18$ & 1.25 & $6 / 9^{+2}$ & $0.63^{+2}$ & $6 / 9$ & 0.63 \\
\hline \#7A+6 D & $6 / 60$ & 4.0 & $6 / 24$ & 1.60 & $6 / 36$ & 2.5 \\
\hline \#7A+7 D & $6 / 60^{-1}$ & $4.0^{-1}$ & $6 / 36$ & 2.5 & $6 / 36$ & 2.5 \\
\hline \#7A+8 D & LNV & LNV & $6 / 60^{-2}$ & $4.0^{-2}$ & LNV & LNV \\
\hline \#7A+9 D & LNV & LNV & LNV & LNV & LNV & LNV \\
\hline \#7A+10 D & LNV & LNV & LNV & LNV & LNV & LNV \\
\hline
\end{tabular}


Table 3. Effect of positive lenses on VA for Subject 1 viewing a Lighthouse near acuity chart at $40 \mathrm{~cm}$ without a pinhole and through a pinhole with a $1.5 \mathrm{~mm}$ and $2 \mathrm{~mm}$ diameter respectively while the target was illuminated at 1 lux. Table 3 . Effect of positive lenses on VA for Subject 1 viewing a Lighthouse near acuity chart at $40 \mathrm{~cm}$ without a pinhole and through a pinhole with a $1.5 \mathrm{~mm}$ and 2 mm diameter respectively while the target was illuminated at 1 lux.

\begin{tabular}{|l|l|l|l|l|l|l|}
\hline Lenses & $\begin{array}{l}\text { VA without pin- } \\
\text { hole }\end{array}$ & M Notation & $\begin{array}{l}\text { VA with pinhole } \\
1.5 \mathrm{~mm}\end{array}$ & M Notation & $\begin{array}{l}\text { VA with pinhole } \\
2 . \mathrm{mm}\end{array}$ & M Notation \\
\hline \# 7A & $6 / 6$ & 0.40 & $6 / 6$ & 0.40 & $6 / 6$ & 0.40 \\
\hline$\# 7 \mathrm{~A}+1 . \mathrm{D}$ & $6 / 7.5$ & 0.50 & $6 / 7.5^{-2}$ & $0.50^{-2}$ & $6 / 9$ & 0.63 \\
\hline$\# 7 \mathrm{~A}+2 . \mathrm{D}$ & $6 / 7.5$ & 0.50 & $6 / 12^{+2}$ & $0.8^{+2}$ & $6 / 12$ & 0.80 \\
\hline$\# 7 \mathrm{~A}+3 . \mathrm{D}$ & $6 / 9^{-2}$ & $0.63^{-2}$ & $6 / 24$ & 1.6 & $6 / 24^{+1}$ & $1.6^{+1}$ \\
\hline$\# 7 \mathrm{~A}+4 . \mathrm{D}$ & $6 / 36$ & 2.5 & $6 / 60$ & 4.0 & $6 / 60^{-2}$ & $4.0^{-2}$ \\
\hline$\# 7 \mathrm{~A}+5 . \mathrm{D}$ & $6 / 60^{-2}$ & $4.0^{-2}$ & LNV & LNV & LNV & LNV \\
\hline \# 7A+6.D & LNV & LNV & LNV & LNV & LNV & LNV \\
\hline
\end{tabular}

Table 4. Effect of negative lenses on VA for Subject 1 viewing a Lighthouse near acuity chart at 40 cm without a pinhole and through a pinhole with a $1.5 \mathrm{~mm}$ and $2 \mathrm{~mm}$ diameter respectively while the target was illuminated at 460 lux.

\begin{tabular}{|l|l|l|l|l|l|l|}
\hline Lenses & $\begin{array}{l}\text { VA without pin- } \\
\text { hole }\end{array}$ & M Notation & $\begin{array}{l}\text { VA with pinhole } \\
1.5 \mathrm{~mm}\end{array}$ & M Notation & $\begin{array}{l}\text { VA with pinhole } \\
\text { 2. mm }\end{array}$ & M Notation \\
\hline \#7A & $6 / 6$ & 0.40 & $6 / 6$ & 0.40 & $6 / 6$ & 0.40 \\
\hline \#7A-1 D & $6 / 6$ & 0.40 & $6 / 6$ & 0.40 & $6 / 6$ & 0.40 \\
\hline \#7A-2 D & $6 / 6$ & 0.40 & $6 / 6$ & 0.40 & $6 / 6$ & 0.40 \\
\hline \#7A-3 D & $6 / 6$ & 0.40 & $6 / 6$ & 0.40 & $6 / 6$ & 0.40 \\
\hline \#7A-4 D & $6 / 6$ & 0.40 & $6 / 6$ & 0.40 & $6 / 6$ & 0.40 \\
\hline \#7A-5 D & $6 / 6$ & 0.40 & $6 / 6^{-2}$ & $0.40^{-2}$ & $6 / 6$ & 0.40 \\
\hline \#7A-6D & $6 / 6$ & 0.40 & $6 / 9^{+2}$ & $0.63^{+2}$ & $6 / 7.5$ & 0.50 \\
\hline \#7A-7 D & $6 / 6^{-2}$ & $0.40^{-2}$ & $6 / 9^{-2}$ & $0.63^{-2}$ & $6 / 9^{-1}$ & $0.63^{-1}$ \\
\hline \#7A-8 D & $6 / 7.5$ & 0.50 & $6 / 18$ & 1.25 & $6 / 18^{+1}$ & $1.25^{+1}$ \\
\hline \#7A-9.00 & $6 / 7.5$ & 0.50 & $6 / 60$ & 4.0 & $6 / 60$ & 4.0 \\
\hline \#7A-10.00 & 6.9 & 0.63 & $6 / 60$ & 4.0 & $6 / 60$ & 4.0 \\
\hline
\end{tabular}

Table 5. Effect of negative lenses on VA for Subject 1 viewing a Lighthouse near acuity chart at $40 \mathrm{~cm}$ without a pinhole and through a pinhole with a $1.5 \mathrm{~mm}$ and $2 \mathrm{~mm}$ diameter respectively while the target was illuminated at 1 lux.

\begin{tabular}{|l|l|l|l|l|l|l|}
\hline Lenses & $\begin{array}{l}\text { VA without pin- } \\
\text { hole }\end{array}$ & M Notation & $\begin{array}{l}\text { VA with pinhole } \\
1.5 \mathrm{~mm}\end{array}$ & M Notation & $\begin{array}{l}\text { VA with pinhole } \\
2 . \mathrm{mm}\end{array}$ & M Notation \\
\hline \#7A & $6 / 7.5$ & 0.50 & $6 / 12$ & 0.80 & $6 / 12$ & 0.80 \\
\hline \#7A-1 D & $6 / 7.5$ & 0.50 & $6 / 9^{-1}$ & $0.63^{-1}$ & $6 / 9$ & 0.63 \\
\hline \#7A-2 D & $6 / 7.5$ & 0.50 & $6 / 9^{-2}$ & $0.63^{-2}$ & $6 / 9^{-2}$ & $0.63^{-2}$ \\
\hline \#7A-3 D & $6 / 7.5$ & 0.50 & $6 / 12$ & 0.80 & $6 / 9^{-3}$ & $0.63^{-3}$ \\
\hline \#7A-4 D & $6 / 7.5$ & 0.50 & $6 / 18$ & 1.25 & $6 / 18$ & 1.25 \\
\hline \#7A-5 D & $6 / 9$ & 0.63 & $6 / 24$ & 1.6 & $6 / 24$ & 1.6 \\
\hline \#7A-6 D & $6 / 9$ & 0.63 & $6 / 36$ & 2.5 & $6 / 60$ & 4.0 \\
\hline \#7A-7 D & $6 / 12$ & 0.80 & $6 / 60$ & 4.0 & $6 / 60$ & 4.0 \\
\hline \#7A-8 D & $6 / 60$ & 4.0 & LNV & LNV & LNV & LNV \\
\hline \#7A-9 D & LNV & LNV & LNV & LNV & LNV & LNV \\
\hline
\end{tabular}




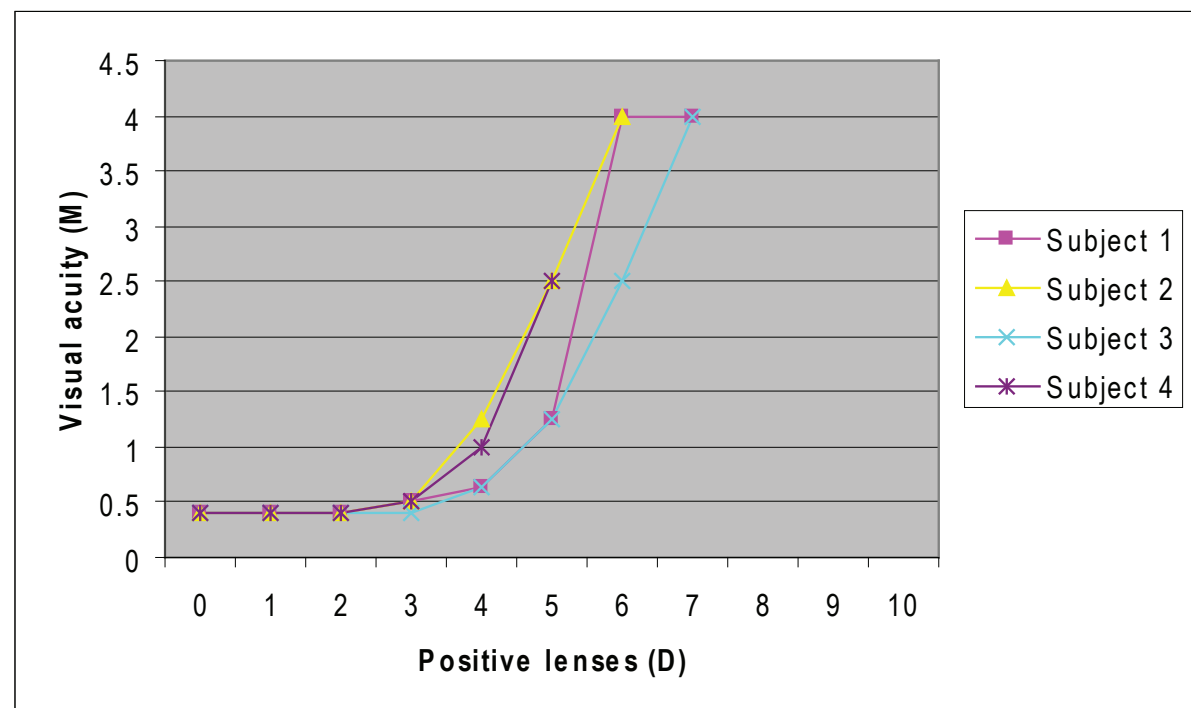

Figure 1. Effect of positive lenses on visual acuity while viewing a near acuity chart at $0.4 \mathrm{~m}$, without a pinhole, illuminated at 460 lux. M notation is used for all plots and on this scale $6 / 6=0.4$ and $6 / 60=4 \mathrm{M}$.

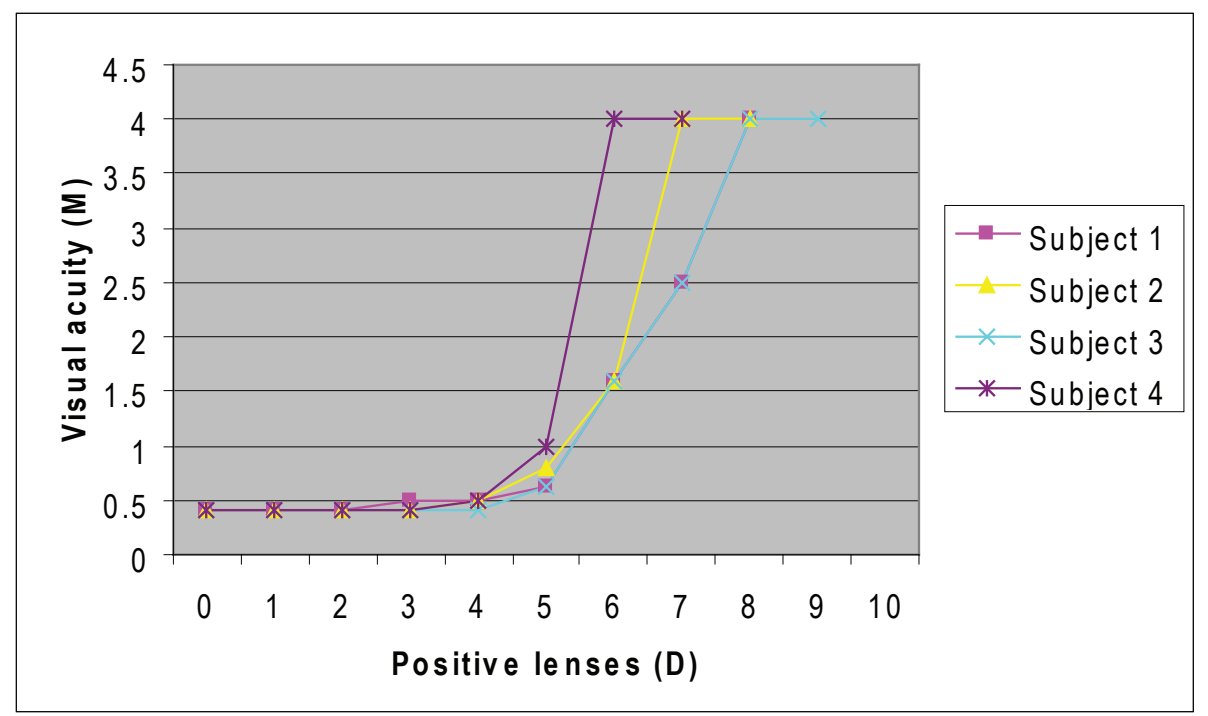

Figure 2. Effect of positive lenses on visual acuity while viewing a near acuity chart at $0.4 \mathrm{~m}$ through a pinhole with a $1.5 \mathrm{~mm}$ diameter and illumination of 460 lux.

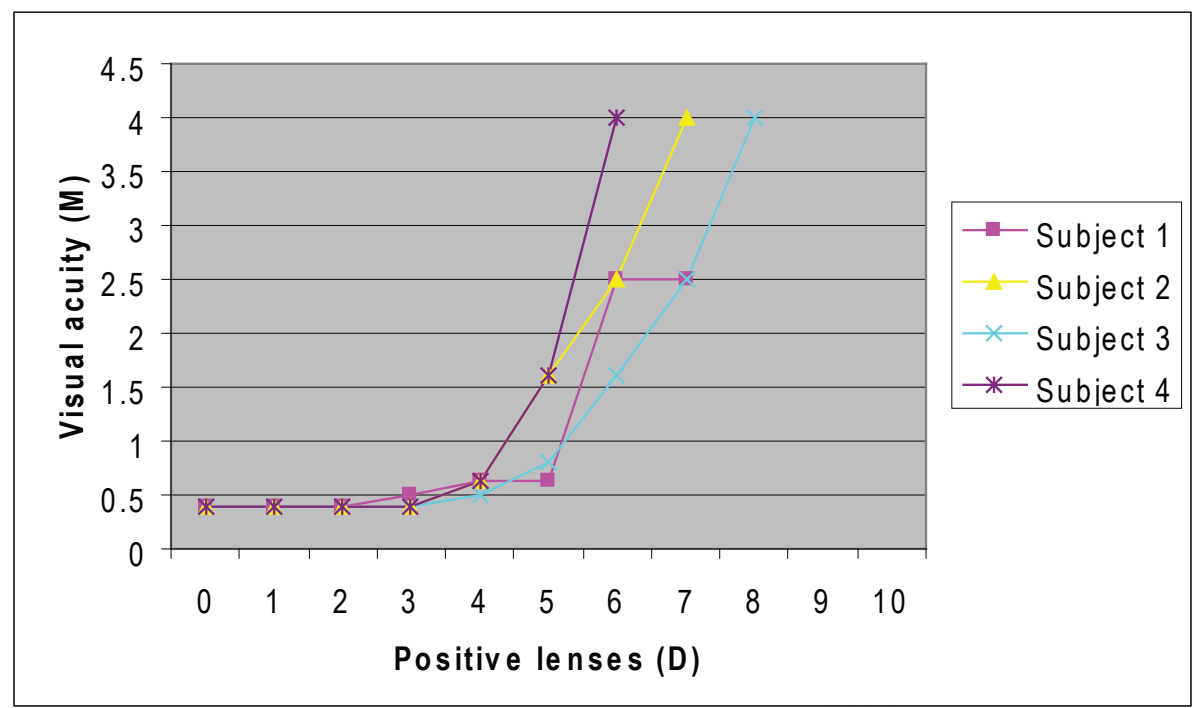

Figure 3. Effect of positive lenses on visual acuity while viewing a near acuity chart at $0.4 \mathrm{~m}$ through a pinhole with a diameter of $2 \mathrm{~mm}$ and illumination of 460 lux.. 


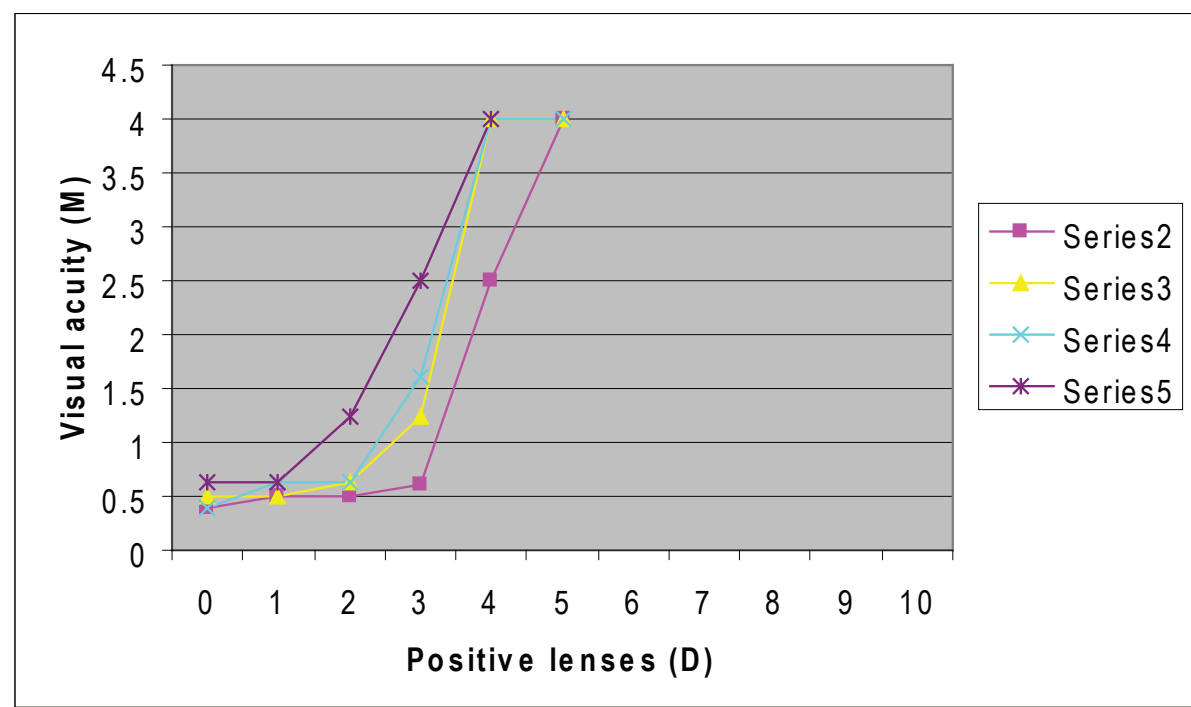

Figure 4. Effect of positive lenses on visual acuity while viewing a near acuity chart at $0.4 \mathrm{~m}$ without a pinhole and illumination of 1 lux.

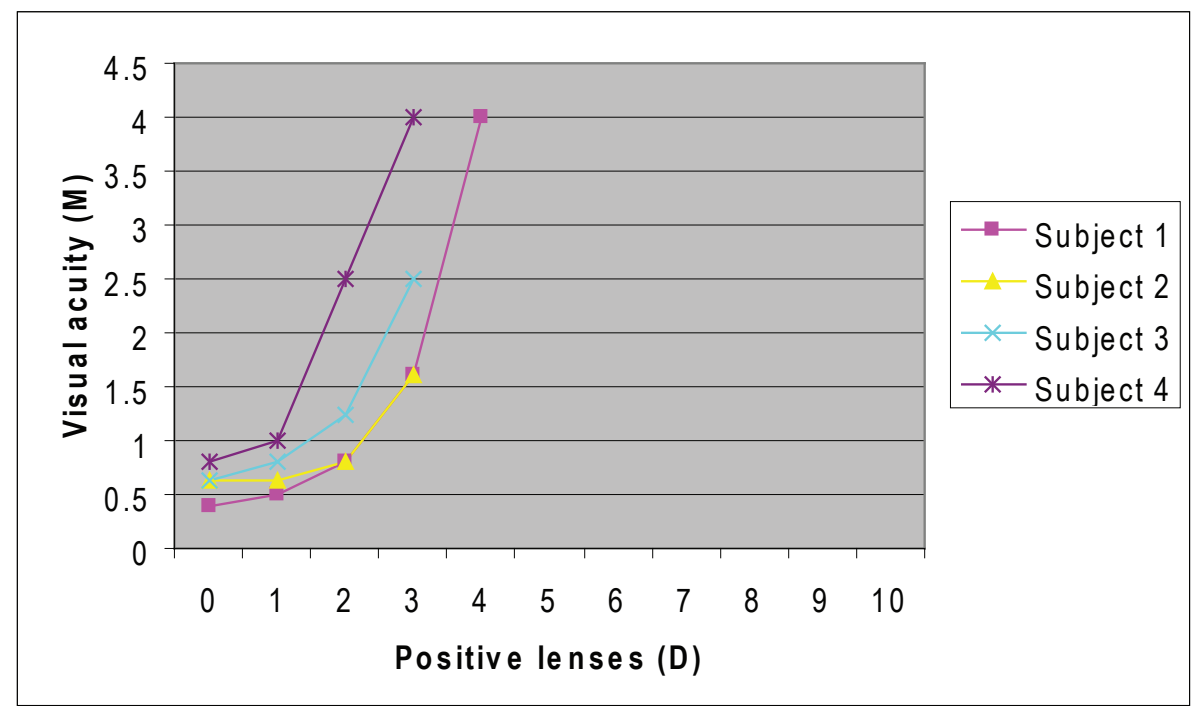

Figure 5. Effect of positive lenses on visual acuity viewing a near acuity chart at $0.4 \mathrm{~m}$ through a pinhole with a $1.5 \mathrm{~mm}$ diameter and illumination of 1 lux.

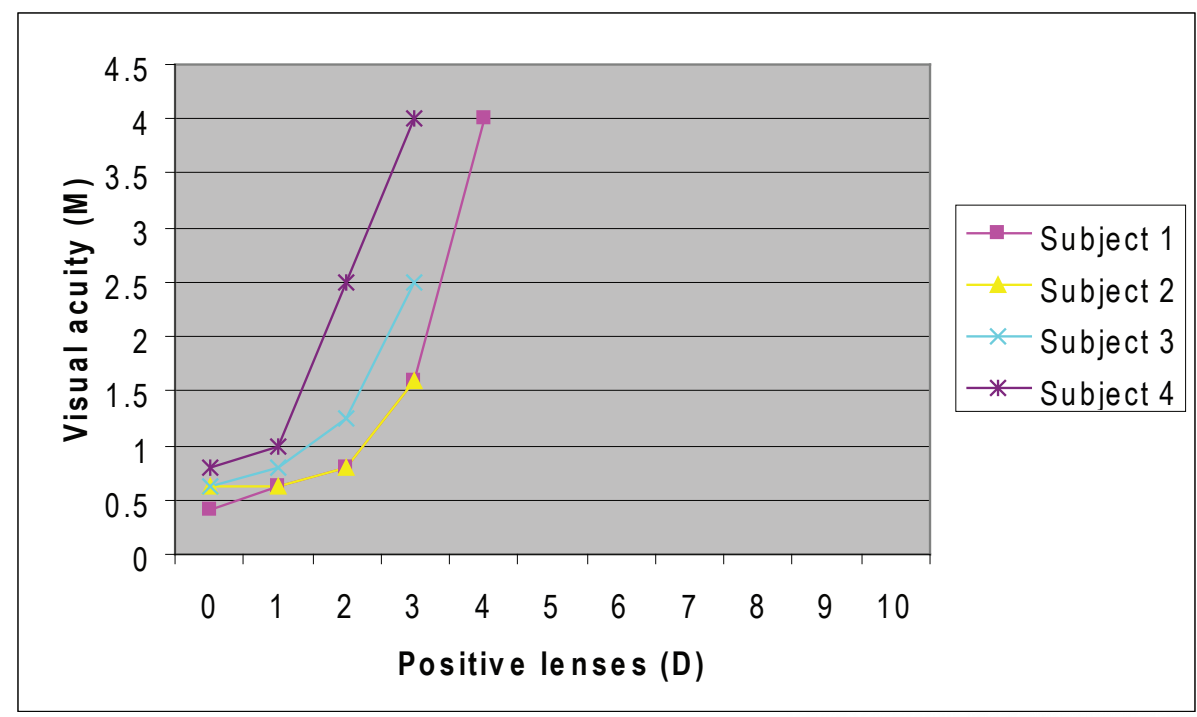

Figure 6. Effect of positive lenses on visual acuity viewing a near acuity chart at $0.4 \mathrm{~m}$ through a pinhole with a $2 \mathrm{~mm}$ diameter and illumination of 1 lux. 


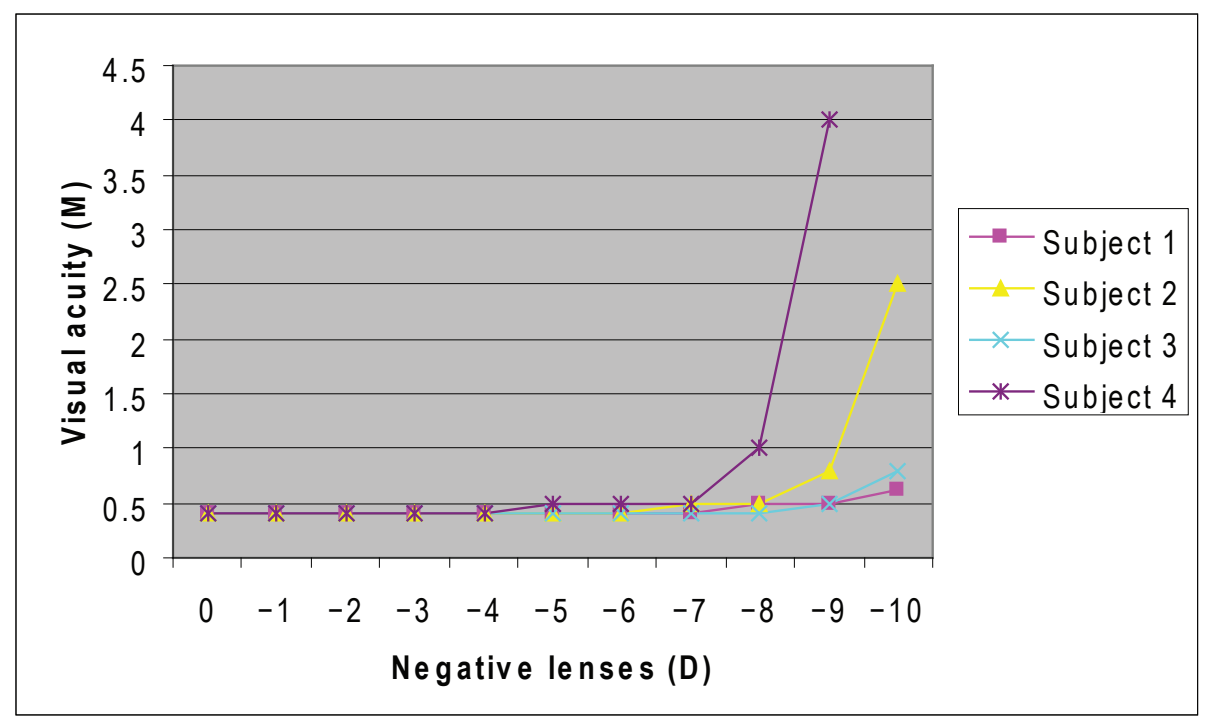

Figure 7. Effect of negative lenses on visual acuity while viewing a near acuity chart without a pinhole and illumination of 460 lux.

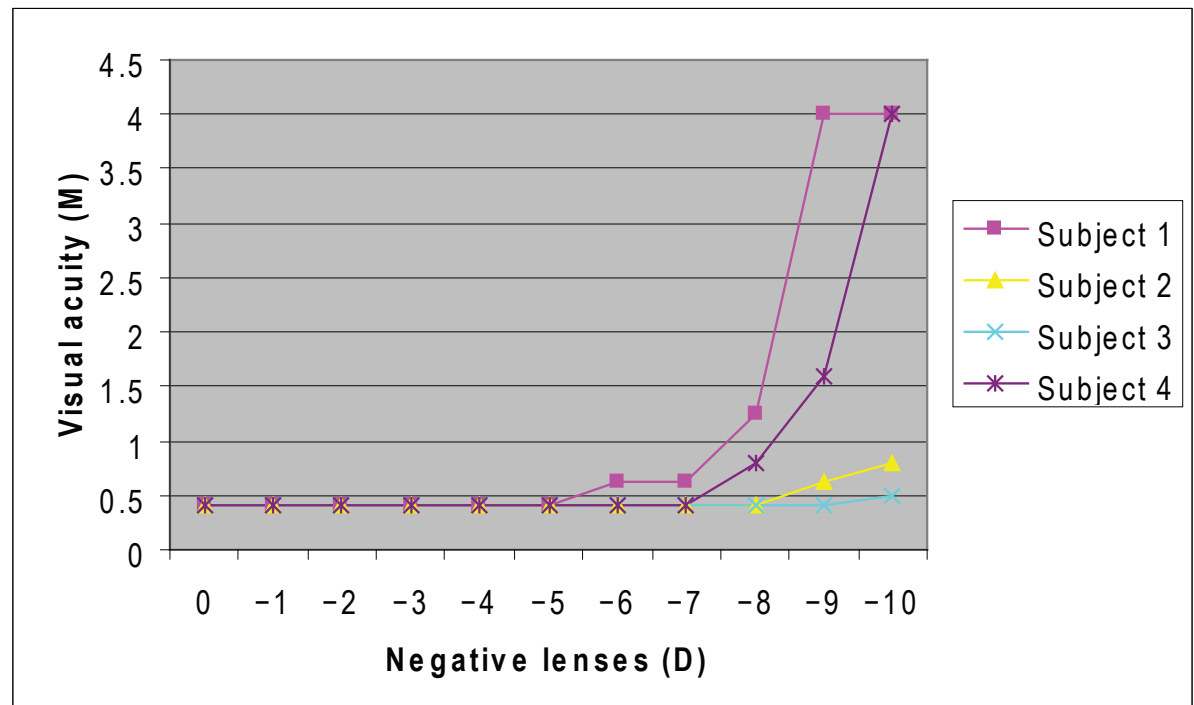

Figure 8. Effect of negative lenses on visual acuity while viewing a near acuity chart at $0.4 \mathrm{~m}$ through a pinhole with a $1.5 \mathrm{~mm}$ diameter and illumination of 460 lux.

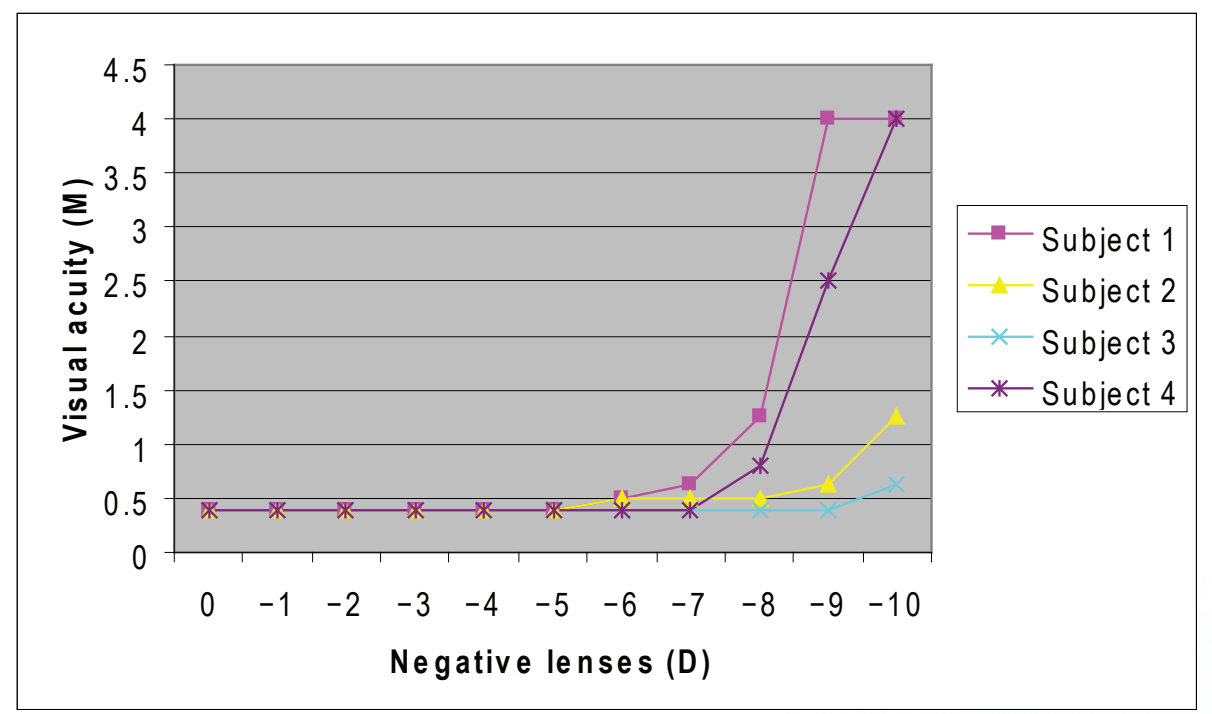

Figure 9. Effect of negative lenses on visual acuity while viewing a near acuity chart at $0.4 \mathrm{~m}$ through a pinhole with a $2 \mathrm{~mm}$ diameter and illumination of 460 lux. 


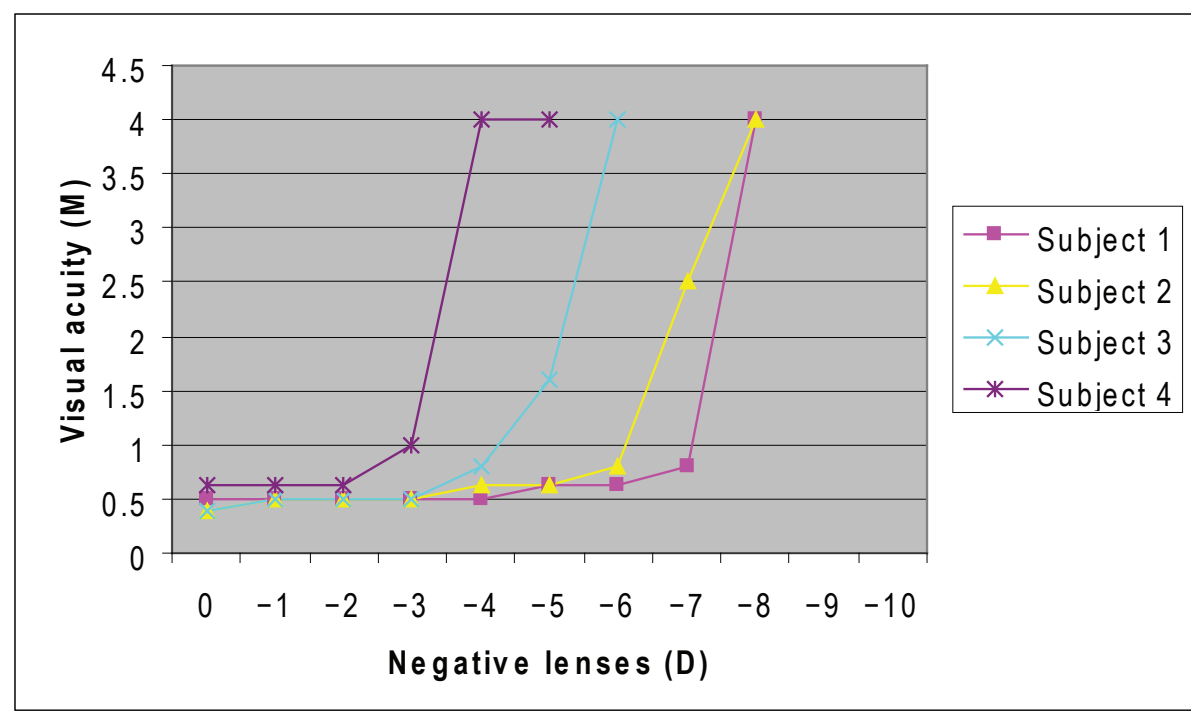

Figure 10. Effect of negative lenses on visual acuity while viewing a near acuity chart at $0.4 \mathrm{~m}$ without a pinhole and illuminated of 1 lux.

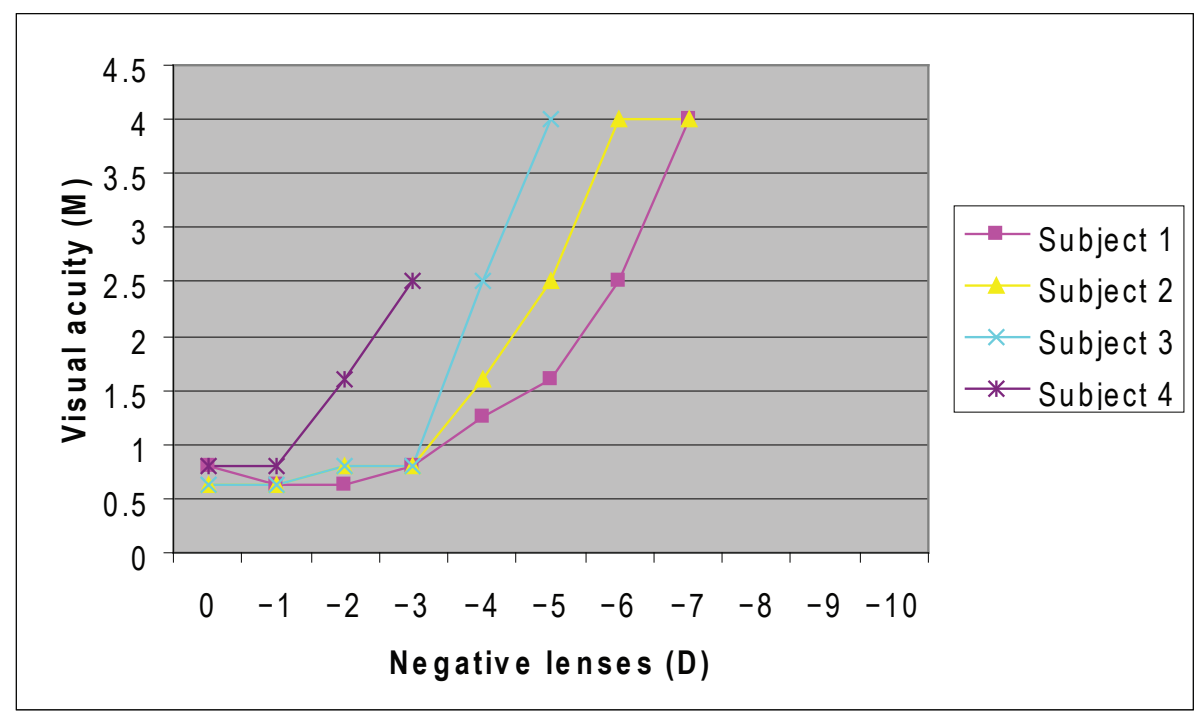

Figure 11. Effect of negative lenses on visual acuity while viewing a near acuity chart at $0.4 \mathrm{~m}$ through a pinhole with a $1.5 \mathrm{~mm}$ diameter and illumination of 1 lux.

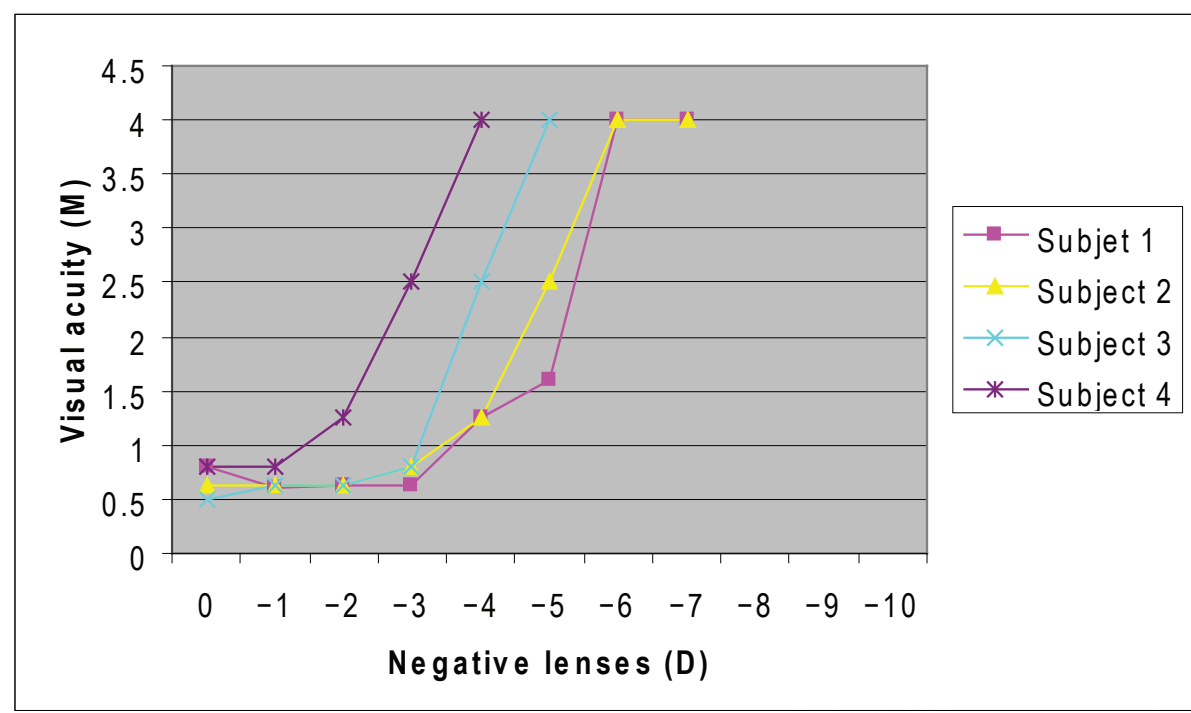

Figure 12. Effect of negative lenses on visual acuity while viewing a near acuity chart at $0.4 \mathrm{~m}$ through a pinhole with a $2 \mathrm{~mm}$ diameter and illumination of 1 lux 
Figures 1 and 7 were used as the reference graphs to facilitate interpretation of our results. Figures 1-6 represent the comparison of the visual acuity (measured in M-notation) and the effect of positive lenses on the relaxation of accommodation (measured in dioptres) while Figures 7 to 12 compared visual acuity and the effect of negative lenses on the stimulation of accommodation. When the illumination was high (460 lux) and a pinhole was added it was established that in all of the cases the visual acuity remained stable for much longer and further lenses were needed to cause a reduction in visual acuity. As soon as illumination was decreased to 1 lux there was a dramatic change in visual acuity as well as the accommodative response. The VA already started to decrease when low powered lenses were added while at the same point (with the same lens) there was no major change when the illumination was much higher.

When looking closely at Figures 1 to 3 it can be observed that the pinhole actually allowed the vision to be more stable and thus more positive lenses could be added before a reduction in VA was eventually seen. There was one factor, however, that remained constant although the illumination was changed and that was the degree of VA reduction that took place. As soon as the accommodative threshold was reached the VA reduction pattern was found to be similar in all three graphs. As mentioned previously, low illumination caused a reduction in the amount of positive power that could be accepted at near and VA was already reduced to $6 / 60$ when only $4 \mathrm{D}$ was added in most of the cases while as can be seen in Figure 1 at least $1 \mathrm{D}$ extra could be added before VA was 6/60.

When the pinholes were introduced even less positive lenses were needed to reduce VA (as seen when Figure 4 is compared to Figures 5 and 6). Nevertheless, this reduction was not drastic. If we compare Figures 2 and 3 with Figures 5 and 6 we note that only about $1 \mathrm{D}$ was needed to reduce the VA while in low illumination whereas in the higher illumination up to $4 \mathrm{D}$ were added successfully before VA was affected.

As expected Figure 7 showed that much more negative lenses could be added to reduce VA than positive lenses which correlated with the subject's larger amplitudes of accommodation. The comparison between Figures 7 and 10 showed similar results to those seen, when Figures 1 and 4 were compared. Low illumination produced a reduction in VA when lenses of power $-3 \mathrm{D}$ were added while in high illumination conditions up to $-7 \mathrm{D}$ could be added before
VA was really affected. The pinhole actually reduced accommodative response as well as the VA when illumination was reduced. With the exception of Subject 1 greater powered negative lenses were required to reduce the VA significantly. There was an improvement in VA in all subjects when the pinholes were introduced in high illumination. This can be seen by comparing Figures 8 and 9 to Figure 7 . Figures 11 and 12 showed slight differences when compared with Figure 10. Subjects 3 and 4 showed slight reduction in the amount of negative lenses needed to reduce VA. However, the reduction followed a similar pattern in all three figures.

Lastly we looked at the effect of the different pinhole size diameters. When viewing Figure 3 where the larger pinhole was used there was very little change compared to Figure 2. The change was so small it could be considered to be insignificant. Similar results were found when Figures 5 and 6 were compared. With the lenses of negative power there were more noticeable improvements in the VA while a pinhole was in place, but they were not significant. In Figure 9, for Subjects 2 and 3, VA reduction was larger when the $-11 \mathrm{D}$ lenses were added and this could be attributed to the fact that all the available accommodation was utilized. This power, however, did fall out of our test range. When Figure 11 is compared to Figure 10 Subjects 2, 3 and 4 showed similar results in both figures with the exception being Subject 1 who had a VA of $6 / 60$ with -6 D instead of $-7 \mathrm{D}$.

\section{The effect of pinholes on depth of focus}

When light from a distant or near object enters the uncompensated eye, the area of the pupil is filled with light rays from each point of the object. These rays are then refracted and they may focus in front, on or behind the retina. If the ray focuses on the retina a clear image will be formed. If the rays focus in front of the retina, they will continue to diverge to the retina and form a blurred circular (because of the circular shape of the pupil) patch on the retina commonly known as a blurred circular patch or blurred circle. The size or amount of blur is determined by the size the marginal rays of the diverging rays (or pencil) are on the retina. If the rays focus behind the retina, the size of the blurred circular image is also determined by the size the marginal rays are in the convergent pencil incident on the retina. This is shown in Figure 13. In this case the subject may accommodate, thus reducing the blurred circular image and form a clear image 
on the retina. When we do a normal ocular refraction on an eye, the compensating lenses refract the light to form a clear image on the retina. If the refraction is not correct (over-minused or over-plussed) the image cone of rays will not form an image point on the retina and the sequential blur will be in proportion to the diameter of the marginal rays that enter the retina. The lens that one prescribes will lessen the diameter of the blur circles according to how efficient one's optical refraction is. The pinhole likewise reduces the blur circles. When the patient wears this optical compensation, although it is imperfect, and it improves the patient's acuity, the pinhole over the prescription will give better results by increasing the depth of focus than when the pinhole is just placed before the na- ked eye. This is because the prescription lenses have already contributed to changing the slant of the image rays. In more simple terms, any object or scene that we see is reflecting light in all directions. We can say that the pinhole restricts which light rays can pass to a certain area. When the pinhole fails to improve vision, it indicates that the vision cannot be improved due to functional defect or structural change ${ }^{1}$.

Results obtained from our study concur with two previous studies, namely by Borish ${ }^{1}$ and Loewestein et $a l^{2}$. The latter stated that there was an improvement in visual acuity when a pinhole disc was placed in front of the subject during a visual screening. From our comparisons between Figures 1 and 2 and Figures 7 and 8 they showed a more stable VA and more

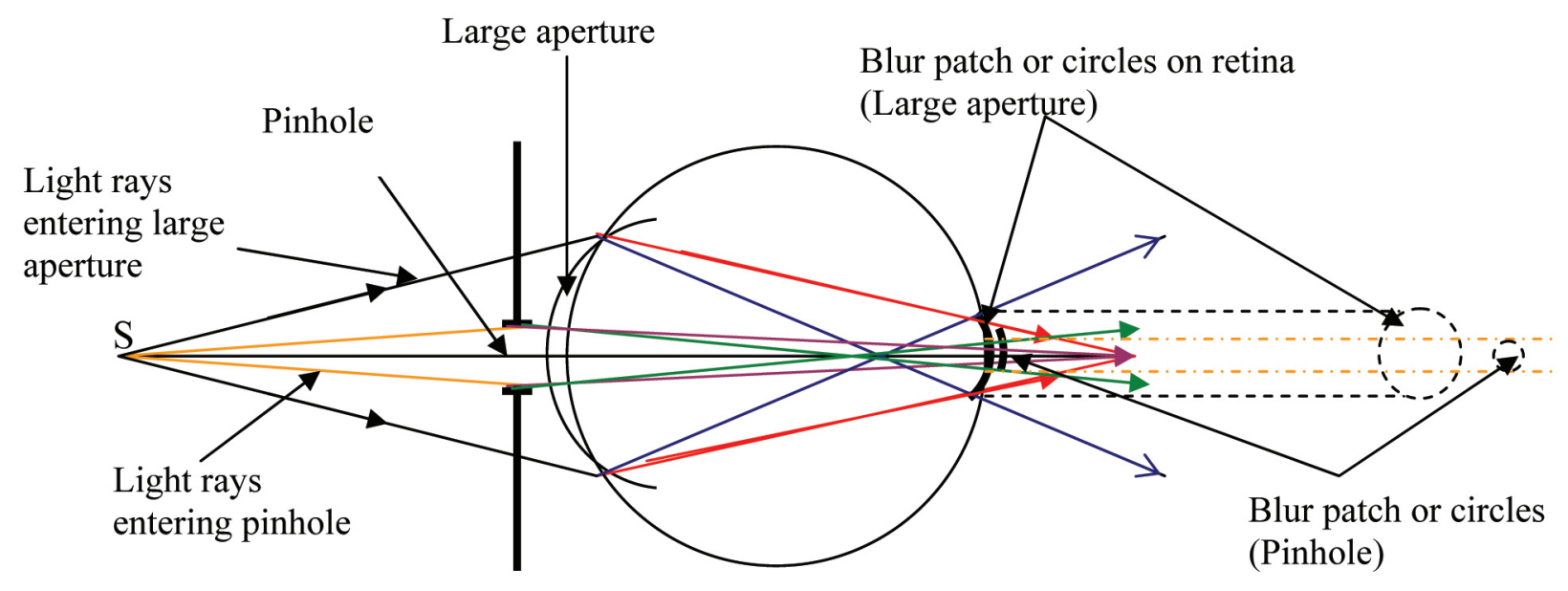

Figure 13. Light from a point source $S$ enters the eye, firstly through a large circular aperture (black lines). After refraction the converging pencil may form a focus in front of or behind the retina. If in front (blue lines) the pencil continues to diverge away from the point focus towards the retina and forms a blur patch or circle on the retina. The shape of the blur patch is a result of the aperture being circular in shape. The size of the blur is determined by the marginal rays of the pencil that reach the retina. The same will apply if the pencil, after refraction forms a focus behind the retina (red lines). It can be seen that if a smaller aperture is placed in front of the eye (pinhole), the marginal rays of the pencil after refraction form a smaller blur patch on the retina thus reducing the blur (green and purple lines) and thus increasing the depth of focus.

lenses were needed to cause a VA reduction. Borish ${ }^{1}$ looked at macular lesions and impaired light sense problems and found that a pinhole does not improve the VA, but actually causes a reduction in VA. When comparing Figures 5 and 6 with 4, 11 and 12 to graph 10 the same results were found. As soon as a pinhole, regardless of its diameter, was placed in front of a subject in low illumination we noticed that much less lenses were needed to cause a reduction in VA. A possible explanation for this phenomenon is that in low illumination the pinhole removes the majority of this illumination ensuring that less light reaches the retina and effectively reduces the VA. This causes the light intensity to drop and the letters became less distinct and more difficult to read.

One of the main reasons for this research project was to investigate whether pinholes with various diameters had diverse effects on visual acuity or not. From our comparisons we observed that the difference in VA between a $1.5 \mathrm{~mm}$ and a $2 \mathrm{~mm}$ diameter pinhole is really insignificant when looking at VA improvement and even with VA reduction caused by low illumination there were no major differences in the results of the various sized pinholes.

Possible strengths in this study were the following: 
- Young patients were selected because of their large accommodative amplitudes to show the effect of accommodation on spherical aberration neu tralization with the pinhole.

- The subjects in this study had fully compensated refractive errors. The reason for this is that a small or reduced pupil size can result in a higher uncompensated $\mathrm{VA}^{10}$.

- One examiner was responsible for obtaining the results from all the subjects to make certain the same testing technique was used throughout.

The possible limitations of the study were that:

- The sample size of four eyes was possibly too small. A larger sample may have given different results.

- Because of the length of the whole procedure, fatigue may have contributed to some of the variations in our findings.

- Only pinhole diameters of $1.5 \mathrm{~mm}$ and $2 \mathrm{~mm}$ were used and perhaps larger or smaller diameter pinholes should have been used.

- Subjects that showed better results than the rest of the group may not have achieved this due to better VA, but rather perhaps due to memorizing the VA chart.

\section{Conclusion}

When we view a proximal target activation of the near synkinesis occurs: accommodation takes place, depression of gaze occurs, the pupils constrict and the eyes converge and incyclorotate. The reason for these changes is to allow the eye to obtain a clear image of the target presented. Pupil constriction, which is an integral part of the near synkinesis, increases the depth of focus and thus reduces the amount of accommodation required for producing a clear image on the retina. By increasing the depth of focus of the eye the range of focusing is increased allowing errors to be eradicated and the image to still have a noticeable amount of clarity. The use of pinholes increases the depth of focus and moreover, reduces the amount of spherical aberration in the human eye.

The pinhole effect appears to offer improvement in VA provided no pathology is present, by reducing the amount of blur presented on the retina. This was evident in our study in which all subjects had an improvement in VA of at least two lines in high illumination. This increase in VA was also evident when accommodation was stabilized (see Figure 1) thus explaining the process whereby pupil constriction also relieves the amount of accommodation required. The sizes of the pinholes used (1.5 and $2 \mathrm{~mm})$, however, did not appear to show any significant differences in the results thus indicating that these pinhole diameters appeared to increase the VA equally. However, this was not the case when illumination was decreased. The proposed reason for this was that in order to 'see', light has to reach the retina and in the scenarios with reduced illumination and adding a pinhole only succeeded in reducing the amount of light available to the retina even more thereby effectively decreasing the VA.

One shortcoming, as previously mentioned was that a greater difference in pinhole diameters should have been used whereby a significant difference in pinhole sizes may have shown different results. Future research using, say, a $0.5 \mathrm{~mm}$ and 1.5 or $2 \mathrm{~mm}$ pinhole under different lighting conditions might be useful.

\section{Acknowledgements}

I would like to thank Ansunel de la Rey for her valuable input and Hester Carlson for her comments.

\section{References}

1. Borish IM. Borish Clinical Refraction. New York: Press books, 1998.

2. Loewenstein JI, Palmberg PF, Connett JE, Wentworth DN. Effectiveness of a pinhole method for visual acuity screening. Archives of Ophthalmology 19852103.

3. Naidoo K. Case finding in the clinic: Refractive errors. Journal of Community Eye Health 200243 39-40.

4. Lou X, Kymes SM, Gordon MO, Bassnett S. Lens fluorescence and accommodative amplitude in pre-presbyopic and presbyopic subjects. Experimental Eye Research 200784 1013-1017.

5. Abolmaali A, Schachar RA, Le T. Sensitivity study of human crystalline lens accommodation. Computer Methods and Programs in Biomedicine 200785 77-90.

6. Atchison D.A, Charman W.N, Woods R.L, Subjective Depth-of-Focus of the Eye. Optometry and Vision Science 75 511-520.

7. Vasudevan B,Ciuffreda K.J, Wang B, Graefe's Archive for Clinical and Experimental Ophthalmology 244 930-937.

8. Collins M.J, Franklin R, Davis, B.A, Optical considerations in the contact lens correction of infant aphakia. Optometry and Vision Science 70 234-240

9. Kasthurirangan S, Glasser A. Age related changes in the characteristics of the near pupil response. Vision Research 46 1393-1403.

10. Grosvenor T. Primary Care Optometry, 3rd Ed. Boston: Butterworth-Heinemann, 2002 pp 136. 